\title{
Pinocembrin flavanone inhibits cell viability in PC-3 human prostate cancer by inducing cellular apoptosis, ROS production and cell cycle arrest
}

\author{
LINHAI SHAO \\ YAJUN SHAO \\ YU YUAN ${ }^{3, *}$ \\ ${ }^{1}$ Department of Urology, Hanzhong \\ Central Hospital, Shaanxi Province \\ Hanzhong, 723000, China \\ ${ }^{2}$ Department of Ultrasound, Xi'an NO.1 \\ Hospital, Shaanxi Province, Xi'an \\ 710002, China \\ ${ }^{3}$ Department of Ophthalmology, \\ Hanzhong Central Hospital, Shaanxi \\ Province, Hanzhong, 723000, China
}

\begin{abstract}
The main purpose of the present study was to evaluate the antitumor effects of pinocembrin in human prostate cancer cells (PC-3) along with investigating its effects on cell apoptosis, endogenous ROS production and cell cycle. MTT assay and clonogenic assays were used to study the effects on cell viability and cancer colony formation, respectively. Fluorescence microscopy along with Western blotting was used to study apoptotic effects induced by pinocembrin. Flow cytometry was used to study effects on ROS production and cell cycle phase distribution. Results indicated that pinocembrin promoted inhibition cell proliferation along with reducing cancer colony formation of PC-3 cells in a dose-dependent manner. Pinocembrin induced regulatory effects over expressions of caspase-3, caspase-9, Bax and Bcl-2, thereby promoting apoptotic cell death in PC-3 cells. It also led to the dose-dependent G0/G1 cell cycle arrest. In conclusion, pinocembrin exhibits strong anticancer effects in human prostate cancer cells mediated via apoptosis, endogenous ROS production and G0/G1 cell cycle arrest.
\end{abstract}

Keywords: prostate cancer, flavones, pinocembrin, apoptosis, cell cycle arrest

Pinocembrin or 5,7-dihydroxyflavanone is an active flavone molecule with remarkable therapeutic potential like other flavones (apigenin, kaempferol and chrysin) (1, 2). Flavones have been identified throughout kingdom Plantae as key secondary metabolites typically belonging to the benzopyran class of natural products $(3,4)$. Flavones disclose both lipophilic and hydrophilic activities including modulatory effects on several cellular enzymes. Other than antimicrobial activities in plants, flavones demonstrate significant biological and pharmacological activities against various metabolic disorders in humans (5). These include anticancer, antimicrobial, antioxidant, vascular, anti-allergic, anti-inflammatory, cytotoxic, antimalarial and antiestrogenic activities (6-8). Moreover, flavones have been used against different metabolic complications like atherosclerosis, Alzheimer's disease, cancer and diabetes (9). Pinocembrin - active flavone, has been isolated from a number of

\footnotetext{
*Correspondence; e-mail: 1352057334@qq.com
} 
plants including Euphorbia hirta Linn., Eriodictyon californicum and Cryptocarya chinensis, and purified using different techniques of chromatography (10-12). This molecule is of significant importance to the pharmaceutical industry due to its multifunctional behaviour. Pinocembrin is reported to possess antioxidant, antimicrobial, anticancer and antiinflammatory properties (13-16). Moreover, the pinocembrin molecule has a broad therapeutic time window against cerebral ischemic injury and acts as a neuroprotective agent. Pinocembrin inhibits the growth and proliferation of different human colon and leukaemia cancer cell lines. Prostate cancer is a harmful and frequently diagnosed non-cutaneous malignancy prevailing among men globally (17). In the year 2017, the United States of America had nearly 3.3 million survivors and nearly 1.7 million new diagnoses related to prostate cancer (18). Besides, the disease often shows slow growth and progression, it ranks third among cancer-related mortality in men. Maximum numbers of patients with prostate cancer are diagnosed at advanced stages due to its asymptotic behaviour; before the accessibility to PSA (prostate-specific antigen) testing. The development of prostate cancer has been linked to a number of risk factors including genetic mutation (BRCA-1 or BRCA-2), family history, older age and sub-Saharan African ancestry (19). Active investigation, radiation therapy and surgery remain key methodologies in the treatment of localized prostate cancer. Androgen-deprivation therapy is used as a standard of care for newly detected metastatic prostate cancer. Chemotherapy has enhanced survival chances in some risk groups if considered initially. Therefore, to enhance the survival rate in all risk groups the need for innovative therapeutic candidates arises. Keeping in consideration the anticancer activity of the pinocembrin molecule, the current investigation was designed to explore its anticancer potential against prostate cancer PC-3 cells. The effects on apoptosis, endogenous ROS production and cell cycle arrest were also investigated.

\section{EXPERIMENTAL}

\section{Chemicals, reagents, cell culture and conditions}

Following are the chemical reagents and drugs used in the present study. Pinocembrin (HPLC purity $\geq 98 \%$ ), 5-fluorouracil (5-FLU), acridine orange/ethidium bromide, DCFDA (2',7'-dichlorodihydrofluorescein diacetate), Cellular ROS Assay Kit and crystal violet were obtained from Sigma Aldrich (USA). Culturing of the cells was performed within DMEM (Dulbecco's Modified Eagle Medium), purchased from Gibco BRL (USA). Antibodies were purchased from Cell Signalling Technology (USA). The human prostate cancer PC-3 cells were American Type Culture Collection (USA). These cells were cultured within DMEM bearing FBS (Fetal bovine serum) (10 \%), standard antibiotics (penicillin G of concentration $100 \mathrm{U} \mathrm{mL}^{-1}$ and streptomycin of concentration $100 \mu \mathrm{g} \mathrm{mL}^{-1}$ ) and placed under an environment of $5 \% \mathrm{CO}_{2}$ and $95 \%$ air in an incubator at $37^{\circ} \mathrm{C}$.

\section{Viability assays (proliferation and colony formation assessment)}

Vybrant ${ }^{\circledR}$ MTT (3-(4,5-dimethylthiazol-2-yl)-2,5-diphenyl tetrazolium bromide) Cell Proliferation Assay Kit (ThermoFisher Scientific, USA) was used to analyse cell growth of PC-3 cancerous prostate cells after pinocembrin treatment. Briefly, cells were plated onto 96-well plates at $2.5 \times 10^{5}$ cells in each well for $24 \mathrm{~h}$. Thereafter, pinocembrin at variant 
concentrations of $0,6,12,24$ and $48 \mu \mathrm{mol} \mathrm{L}^{-1}$, was supplemented to each well followed by incubation for $12 \mathrm{~h}$ and $24 \mathrm{~h}$, at $37^{\circ} \mathrm{C}$. Treated cells were washed with phosphate-buffered saline (PBS) prior to staining with MTT solution. MTT staining was performed with Vybrant ${ }^{\circledR}$ MTT Cell Proliferation Assay Kit for $4 \mathrm{~h}$ by strictly following the manufacturer's protocol. The addition of MTT solution leads to the formation of formazan crystals which were then dissolved in $500 \mu \mathrm{L}$ of dimethylsulfoxide. Finally, optical density was calculated by recording absorbance at $540 \mathrm{~nm}$ with an ELISA plate reader (HuaMei Inc. Wuhan, China). To estimate cancer colony generation by PC-3 cells, the clonogenic assay was performed. Exponentially growing PC-3 cells were collected and loaded to 6-well plates with 400 cells each well. These well plates were subjected to incubation for $48 \mathrm{~h}$ prior to treatment with variant pinocembrin doses viz. $0,12,24$ and $48 \mu \mathrm{mol} \mathrm{L}^{-1}$. Thereafter, treated cells were left untouched, replacing DMEM after every 3 days, for 12 days. Afterwards, PC-3 cell colonies were washed with PBS followed by fixation in methyl alcohol. Finally, PC-3 colonies were counted under a light microscope (BD-NE610, Shenzhen Boshida Instrument Co., Ltd. (China) and colonies with $\geq 50$ cells were considered significant for counting.

\section{Apoptosis assay}

To examine the apoptosis-inducing potential of the pinocembrin drug, the acridine orange/ethidium bromide (AO/EB) staining assay was performed. In brief, the PC-3 cells were plated onto a 24-well plate at $2 \times 10^{5}$ cells in each well followed by pinocembrin treatment at different doses of $0,12,24$ and $48 \mu \mathrm{mol} \mathrm{L}^{-1}$ for $48 \mathrm{~h}$. Thereafter, pinocembrin treated PC-3 cells were stained with AO/EB staining solution by obeying the manufacturer's protocol. Cancerous PC-3 cells were finally analysed under a magnification power of 200× of FV500 laser scanning confocal microscope (Olympus, Japan).

\section{Flow cytometry}

To monitor different cell-cycle checkpoints, flow cytometric examination of pinocembrin treated PC-3 cells was performed. Exponentially growing PC-3 cells were exposed to the pinocembrin drug with varying doses $0,12,24$ and $48 \mu \mathrm{mol} \mathrm{L}^{-1}$ for $48 \mathrm{~h}$ within 6-well plates. Then were trypsinized using trypsin followed by staining with nuclei staining buffer ( $0.1 \%$ Triton X-100, $3.8 \mathrm{mmol} \mathrm{L}^{-1}$ sodium citrate, PI (propidium iodide) solution (50 $\mu \mathrm{g} \mathrm{mL}^{-1}$ ), and RNase $\mathrm{B}\left(7 \mathrm{kU} \mathrm{mL}{ }^{-1}\right)$ for $2 \mathrm{~h}$. The cells at different cell cycle checkpoints were measured with the help of the Becton-Dickinson FACScan cytofluorometer (USA).

\section{Measurements of reactive oxygen species (ROS)}

The DCFH-DA staining was used to estimate ROS production in pinocembrin treated PC-3 cancerous prostate cells. PC-3 cells were cultured under a humidified atmosphere of $5 \% \mathrm{CO}_{2}$ and $95 \%$ air within a DMEM for $24 \mathrm{~h}$. These cells were harvested and collected for pinocembrin treatment with different concentrations viz. $0,12,24$ and $48 \mu \mathrm{mol} \mathrm{L}^{-1}$ for $48 \mathrm{~h}$. Pinocembrin-treated cells were washed using PBS and then resuspended in $500 \mu \mathrm{L}$ of $10 \mu \mathrm{mol} \mathrm{L}{ }^{-1}$ DCFH-DA for 30 min in dark. Finally, cells were analysed at an emission of $\sim 520 \mathrm{~nm}$ and excitation of $\sim 485 \mathrm{~nm}$ through FACSCanto II Flow Cytometer (BD Biosciences, USA). 


\section{Western blotting assay}

The PC-3 cancerous prostate cells were harvested at $85 \%$ of growth confluence and subjected to pinocembrin treatment at different doses of $0,12,24$ and $48 \mu \mathrm{mol} \mathrm{L}^{-1}$ for $24 \mathrm{~h}$. Treated cells were sampled into different groups and lysed using RIPA (Radioimmunoprecipitation assay) lysis buffer. The amount of protein within each lysate was determined using bicinchoninic acid assay and equal amounts were loaded onto SDS-PAGE for separation followed by transferal to PVDF (polyvinylidene fluoride) membranes. These membranes were blotted with specific primary antibodies (antibodies against Caspase- 3 and -9 , Bcl-2 and Bax proteins) overnight at $4{ }^{\circ} \mathrm{C}$. Thereafter, membranes were treated with HRPconjugated secondary antibodies. Finally, visualization of immunoreactive bands was prepared by using the ImageQuant LAS4000 system (GE Healthcare, USA).

\section{Statistical analysis}

All the data collected from individual experiments were analysed by using GraphPad Prism Demo (version 5; USA) and are expressed as mean \pm SD. The $p<0.05$ and $p<0.01$ were taken as statistically significant. Each experiment for individual drug doses was performed in triplicate.

\section{RESULTS AND DISCUSSION}

\section{Pinocembrin induced antiproliferative effects in PC-3 cells}

Despite cancer treatment going through significant advancements, treatment of prostate cancer remains a big challenge for researchers. The disease remains poorly diagnosed at early stages due to asymptotic behaviour (20). Pinocembrin has been previously reported to suppress the proliferation rate in a number of human cancer cells. In a similar study, pinocembrin attenuated the cell viability of androgen-sensitive LNCaP and androgen-independent PC3 and DU-145 prostate cancer cell lines (21).

Herein, PC-3 cancerous prostate cells were analysed for viability assessment through MTT assay by following the manufacturer protocol. The proliferation rate was significantly retarded after exposing PC-3 cells to different pinocembrin (Fig. 1) doses (0-48 $\mu \mathrm{mol}$ $\left.\mathrm{L}^{-1}\right)$. Pinocembrin induced toxicity enhanced after elongating treatment time from 12 to 24 h. Viability was reduced to almost 40 and $20 \%$ at $48 \mu \mathrm{mol} \mathrm{L}^{-1}$ after 12 and $24 \mathrm{~h}$ of pinocembrin treatment, respectively (Fig. 2a). Pinocembrin showed almost comparable antiproliferative effects against positive controls with 5-fluorouracil (5-FLU) treated cells). Moreover, the antiproliferative effect of pinocembrin against normal non-cancerous cells,<smiles>O=C1CC(c2ccccc2)Oc2cc(O)cc(O)c21</smiles>

Fig. 1. Chemical structure of pinocembrin. 

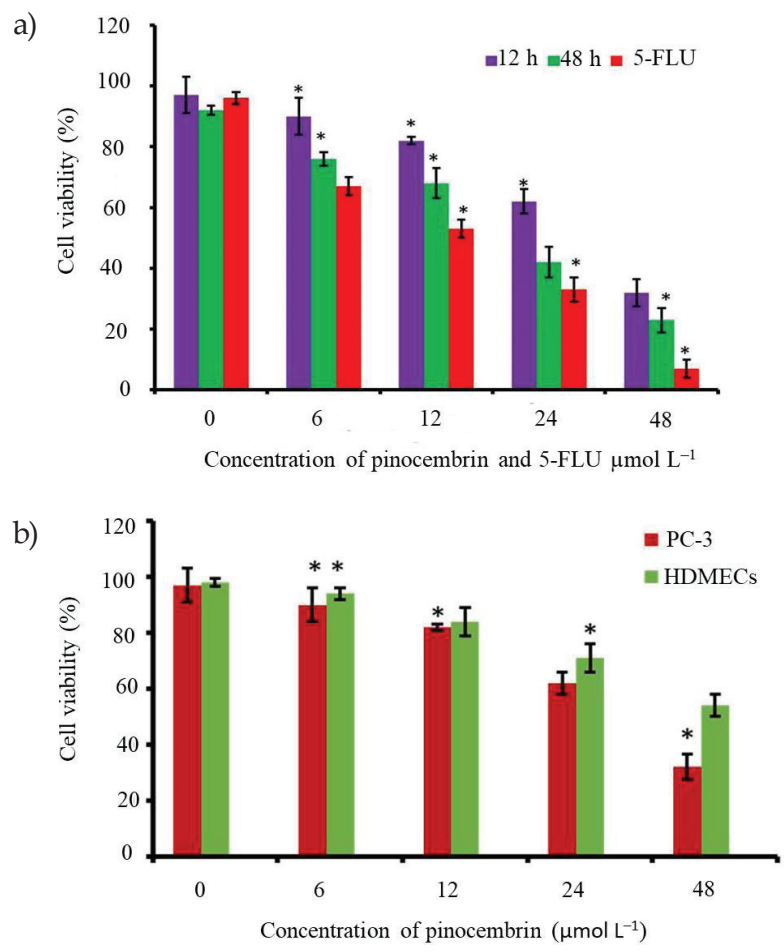

Fig. 2. a) Estimation of the PC-3 cancerous prostate cells viability after pinocembrin treatment at indicated concentration and time intervals. The figure represents the declined number of viable cells after exposure to pinocembrin in comparison to the control group. 5-Fluorouracil (5-FLU) was used as a positive control; b) Effect of pinocembrin on non-cancerous normal cells (human dermal microvascular endothelial cells, HDMECs). Cytotoxicity of pinocembrin was evaluated against these after treatment with the drug for $24 \mathrm{~h}$. The cell viability of these HDMECs after incubation with pinocembrin for $24 \mathrm{~h}$ was much higher than that of similarly treated PC-3 cancer cells. Each experiment was repeated three times and data were presented as mean \pm SD. Statistical significance was taken as ${ }^{*} p<0.05$.

human dermal microvascular endothelial cells (HDMECs) was much lower than PC-3 cells (Fig. 2b). The ability of prostate PC-3 cancer cells to generate and establish colonies was monitored by performing the clonogenic assay. Pinocembrin revealed remarkable toxicity against PC-3 cell colonies (Fig. 3a). Pinocembrin reduced PC-3 colonies to about 100 at 48 $\mu \mathrm{mol} \mathrm{L} \mathrm{L}^{-1}$ in comparison to controls which showed about 400 intact colonies after 12 days of culturing (Fig. 3b). Therefore, the results from MTT assay and colony assessment indicated that pinocembrin induced remarkable cytotoxicity against PC-3 cell proliferation and colony generation.

\section{Pinocembrin caused apoptotic cell death in PC-3 cells}

Apoptosis (natural programmed cell death) is a highly conserved process in mammals and plays a key role in the elimination of damaged, malfunctioning and aged cells. Prevention 
a) $\quad 0 \mu \mathrm{mol} \mathrm{L}-1$

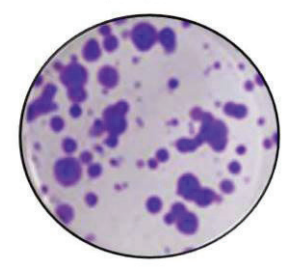

$12 \mu \mathrm{mol} \mathrm{L}-1$
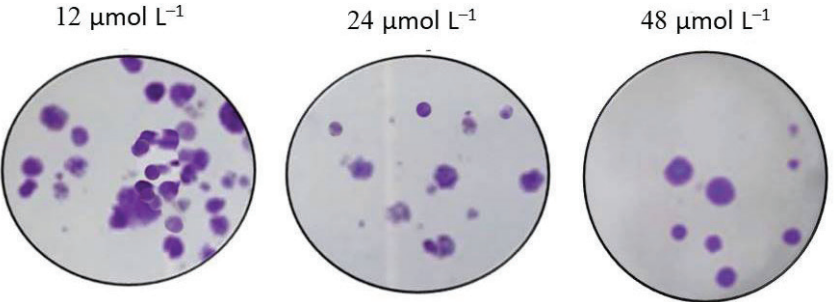

b)

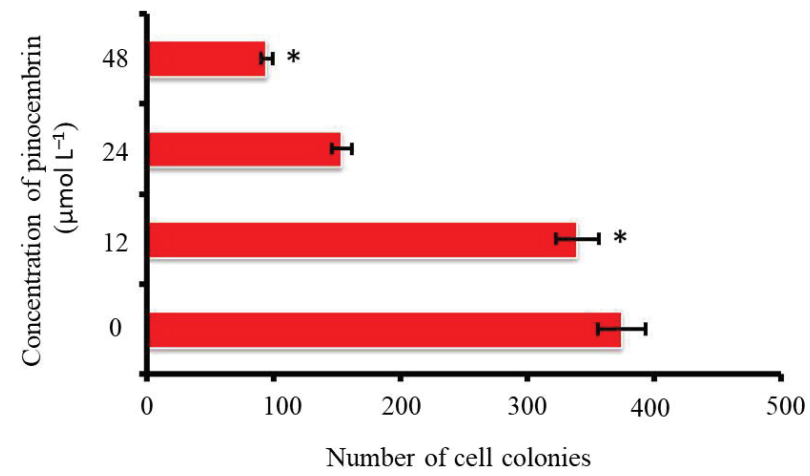

Fig. 3. a) Clonogenic assessment of pinocembrin treated PC-3 cells at variant concentrations; b) Results representing inhibitory effects of pinocembrin at indicated concentrations on PC-3 cells. Each experiment was repeated three times and data are presented as mean \pm SD. The statistical significant figure was taken as ${ }^{*} p<0.05$.

of apoptosis in normal cells transfers them to tumorous cells which then undergo uncontrolled hyper-proliferation (22). Therefore, apoptosis plays a key role in inhibiting the proliferation of cancer cells and serves as a potential target for chemopreventives. Pinocembrin has been shown to promote apoptosis in different human cancers. A study carried out by Zheng et al. has reported that pinocembrin promotes apoptosis via modulation of Bcl-2/Bax ratio and suppresses autophagy in human melanoma cells including A375 and B16F10 (23). In a similar study, pinocembrin has been shown to produce protective effects via the mitochondrial apoptotic pathway in SH-SY5Y neuroblastoma cells against MPP+-induced neurotoxicity (24). Similarly, pinocembrin has been previously reported to induce apoptotic cell death in HT-29 and HCT-116 colon cancer and HL-60 leukaemia cells (25).

Herein, AO/EB staining showed that the normal morphology of PC-3 cells was disturbed after the application of pinocembrin at variant doses. Pinocembrin enhanced the number of apoptotic PC-3 cells with increasing doses. The yellow-green, orange-red and red fluorescent cells indicate the number of early and late apoptotic and necrotic cells respectively (Fig. 4a). Thus AO/EB staining indicated stimulation of apoptotic cell death in PC-3 cells post pinocembrin treatment. Moreover, apoptosis stimulation by pinocembrin was supported by Western blotting at molecular levels. The activity of Caspase-9, Caspase-3and Bax proteins increased and that of Bcl-2 protein decreased with increasing pinocembrin doses (Fig. 4b). Hence, AO/EB staining supported by Western blotting indicated that the antiproliferative effects of pinocembrin could be due to its apoptosis stimulation effects in PC-3 cells. 
a)
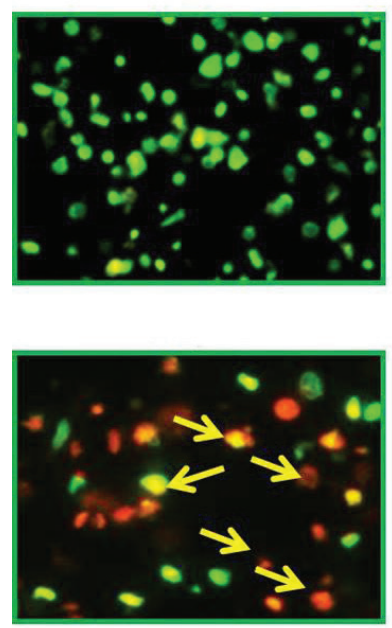

$24 \mu \mathrm{mol} \mathrm{L}-1$
$12 \mu \mathrm{mol} \mathrm{L}-1$
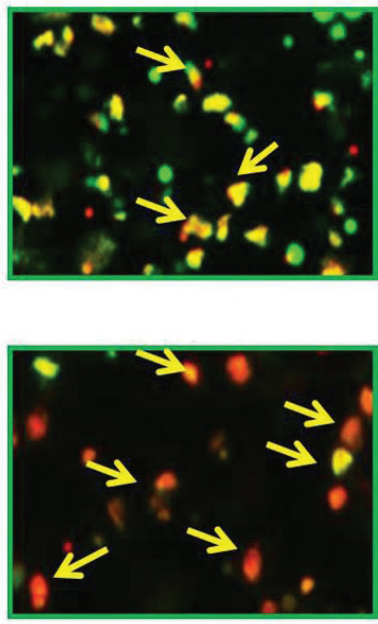

$48 \mu \mathrm{mol} \mathrm{L}-1$

b)

Pinocembrin concentration $(\mu \mathrm{mol} \mathrm{L}-1)$

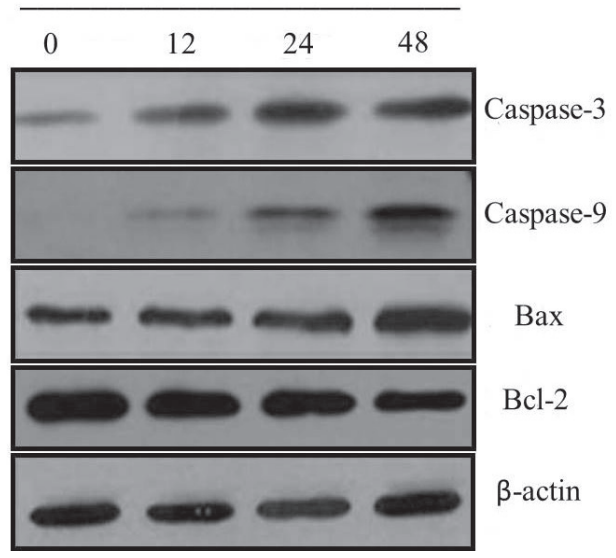

Fig. 4. a) The AO/EB staining assay was implemented for the determination of apoptosis stimulatory potential of the pinocembrin drug in PC-3 cells. The figure shows yellow-green, orange-red and red fluorescence that indicates early, late apoptotic and necrotic cells, respectively; b) Western blotting assay. The figure depicts enhanced expressions of caspases and Bax proapoptotic proteins and lowered Bcl-2 expressions.

\section{Pinocembrin inhibited cell cycle progression in PC-3 cells}

The tendency of hyper-proliferation of cancer cells leads to fast growth and spread of the disease. Therefore, controlling and checking of proliferation rate remains a principal objective of chemopreventives. Pinocembrin has been previously reported to cause G2/M- 


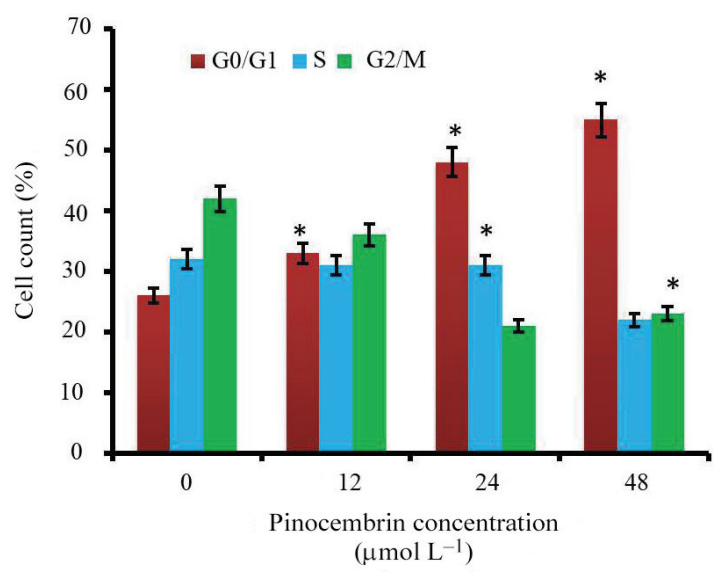

Fig. 5. Analysis of cell cycle phase distribution by flow cytometry after $48 \mathrm{~h}$ of pinocembrin treatment of cancerous PC-3 cells. Pinocembrin triggered cell cycle arrest of cancerous PC-3 cells at the G0/G1 checkpoint in a concentration-dependent manner. Each experiment was repeated three times and data are presented as mean \pm SD. The statistical significant figure was taken as $p<0.05$.

and S-Phase cell cycle arrest in prostate LNCaP cancer cells (21). Herein, flow cytometric measurements were carried out for the estimation of PC-3 cells at different cell cycle checkpoints. Results indicated that the number of cells at $\mathrm{S}$ and $\mathrm{G} 2 / \mathrm{M}$ phases declined with

a)

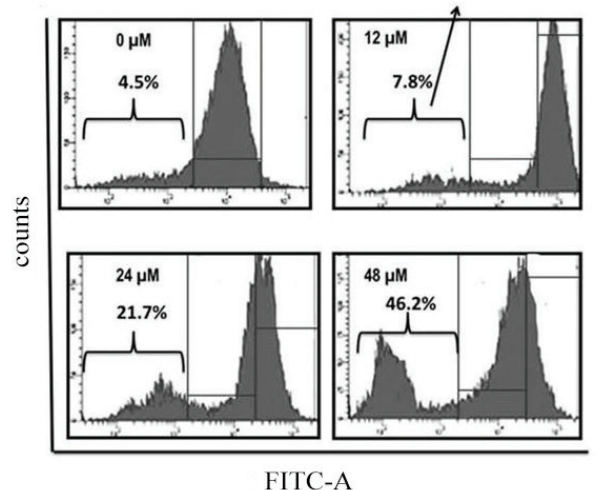

b)

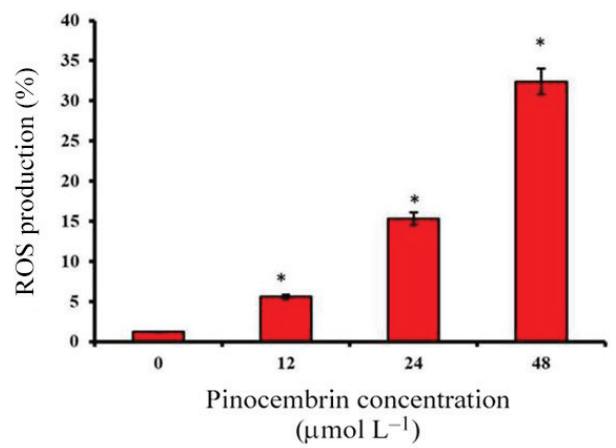

Fig. 6. a) Effects of pinocembrin drug on cancerous PC-3 cells after $48 \mathrm{~h}$ long treatment and then staining with DCFH-DA. Fluorescence intensity of DCFH-DA is directly proportional to ROS production in pinocembrin treated PC-3 cells. Each experiment was repeated three times; b) Graphical representation of the effect of pinocembrin on the ROS production in PC-3 cells, as can be seen, there was a dose-dependent increase in the percentage of cells with damaged cell membranes indicating significant ROS production leading to collapse of mitochondrial membrane potential. Each experiment was repeated three times and data are presented as mean \pm SD. Statistical significance was taken as ${ }^{*} p<0.05$. 
enhanced doses of pinocembrin while the number of cells at G0/G1 cells amplified. The number of G0/G1 cells enhanced to almost $60 \%$ in comparison to about $25 \%$ in the control group (Fig. 5).

\section{Pinocembrin enhanced ROS in PC-3 cells}

ROS are considered lethal entities that disturb a large number of normal cellular processes. As these species are highly reactive they can target any cellular mechanism. Herein, the effects of pinocembrin upon ROS in PC-3 cells were assessed by DCFH-DA staining assay. The results indicated enhanced DCFH-DA fluorescence (directly proportional to ROS) with increasing pinocembrin treatment (Fig. 6a). Therefore, it may be concluded that the antiproliferative effects of pinocembrin are also allied with enhanced ROS. Fig. $6 \mathrm{~b}$ shows the graphical increase in ROS production with an increase in pinocembrin dose.

\section{CONCLUSIONS}

Taken altogether, the results of the current investigation indicate that pinocembrin flavanone exhibited significant antitumor effects against prostate tumor PC-3 cells. These effects were mediated through stimulation of apoptosis, endogenous ROS production and cell cycle arrest.

\section{REFERENCES}

1. G. Zhao, G. W. Qin, J. Wang, W. J. Chu and L. H. Guo, Functional activation of monoamine transporters by luteolin and apigenin isolated from the fruit of Perilla frutescens (L.), Neurochem. Int. 56 (2010) 168-176; https://doi.org/10.1016/j.neuint.2009.09.015

2. J. A. Van Meeuwen, N. Korthagen, P. C. De Jong, A. H. Piersma and M. Van den Berg, (Anti) estrogenic effects of phytochemicals on human primary mammary fibroblasts, MCF-7 cells and their co-culture, Toxicol. Appl. Pharmacol. 221 (2007) 372-383; https://doi.org/10.1016/j.taap.2007.03.016

3. X. Su, L. Kong, X. Lei, L. Hu, M. Ye and H. Zou, Biological fingerprinting analysis of traditional Chinese medicines with targeting ADME/Tox property for screening of bioactive compounds by chromatographic and MS methods, Mini Rev. Med. Chem. 7 (2007) 87-98; https://doi. org/10.2174/138955707779317830

4. T. Y. K. Chan, J. C. N. Chan, B. Tomlinson and J. A. J. H. Critchley, Chinese herbal medicines revisited: a Hong Kong perspective, Lancet 342 (1993) 1532-1534; https://doi.org/10.1016/S01406736(05)80091-1

5. A. K. Verma and R. Pratap, The biological potential of flavones, Nat. Prod. Rep. 27 (2010) 1571-1593.

6. T. Guardia, A. O. Juarez and L. E. Pelzer, Anti-inflammatory properties of plant flavonoids. Effects of rutin, quercetin and hesperidin on adjuvant arthritis in rat, Il Farmaco 56 (2001) 683-687; https:// doi.org/10.1016/S0014-827X(01)01111-9

7. C. H. Jung, C. H. Cho and C. J. Kim, Anti-asthmatic action of quercetin and rutin in conscious guinea-pigs challenged with aerosolized ovalbumin, Arch. Pharm. Res. 30 (2007) 1599-1607; https:// doi.org/10.1007/BF02977330

8. G. B. Reddy, P. Muthenna, C. Akileshwari, V. Saraswat and J. M. Petrash, Inhibition of aldose reductase and sorbitol accumulation by dietary rutin, Curr. Sci. 101 (2011) 1191-1197.

9. J. B. Harborne and C. A. Williams, advances in flavonoid research since 1992, Phytochemistry 6 (2000) 481-504; https://doi.org/10.1016/S0031-9422(00)00235-1 
10. Y. Wu, W. Qu, D. Geng, J. Y. Liang and Y. L. Luo, Phenols and flavonoids from the aerial part of Euphorbia hirta, Chin. J. Nat. Med. 10 (2012) 40-42; http://dx.doi.org/10.3724/SP.J.1009.2012.00040

11. T. H. Chou, J. J. Chen, C. F. Peng, M. J. Cheng and I. S. Chen, New flavanones from the leaves of Cryptocarya chinensis and their antituberculosis activity, Chem. Biodivers. 8 (2011) 2015-2024; https:// doi.org/10.1002/cbdv.201000367

12. Y. L. Liu, D. K. Ho, J. M. Cassady, V. M. Cook and W. M. Baird, Isolation of potential cancer chemopreventive agents from Eriodictyon californicum, J. Nat. Prod. 55 (1992) 357-363; https://doi. org/10.1021/np50081a012

13. X. Lan, W. Wang, Q. Li and J. Wang, The natural flavonoid pinocembrin: molecular targets and potential therapeutic applications, Mol. Neurobiol. 53 (2016) 1794-1801; https://dx.doi. org/10.1007\%2Fs12035-015-9125-2

14. M. A. S. Kumar, M. Nair, P. S. Hema, J. Mohan and T. R. Santhoshkumar, Pinocembrin triggers Bax-dependent mitochondrial apoptosis in colon cancer cells, Mol. Carcinog. 46 (2007) 231-241, https://doi.org/10.1002/mc.20272

15. L. Estevinho, A. P. Pereira, L.Moreira, L. G. Dias and E. Pereira, Antioxidant and antimicrobial effects of phenolic compounds extracts of Northeast Portugal honey, Food Chem. Toxicol. 46 (2008) 3774-3779; https://doi.org/10.1016/j.fct.2008.09.062

16. R. Feng, Z. K. Guo, C. M. Yan, E. G. Li, R. X. Tan and H. M. Ge, Anti-inflammatory flavonoids from Cryptocarya chingii, Phytochemistry 76 (2012) 98-105; https://doi.org/10.1016/j.phytochem.2012.01.007

17. R. L. Siegel and A. Miller Jemal, Cancer statistics, 2017, CA Cancer J. Clin. 67 (2017) 7-30; https://doi. org/10.3322/caac. 21442

18. Surveillance, Epidemiology, and End Results (SEER) Program. Cancer Statistics (2007-2013), Prostate Cancer, Bethesda, MD: National Cancer Institute, USA.

19. S. E. Eggener, A. S. Cifu and C. Nabhan, Prostate cancer screening, JAMA. 314 (2015) 825-826.

20. R. Etzioni, R. Cha, E. J. Feuer and O. Davidov, Asymptomatic incidence and duration of prostate cancer, Am. J. Epidemiol. 148 (1998) 775-785; https://doi.org/10.1097/00005392-199907000-00083

21. Z. Chen, A. Rasul, C. Zhao, F. M. Millimouno, I. Tsuji, T. Yamamura, R. Iqbal, M. Malhi, X. Li and J. Li, Antiproliferative and apoptotic effects of pinocembrin in human prostate cancer cells, Bangladesh J. Pharmacol. 8 (2013) 255-262; https://doi.org/10.3329/bjp.v8i3.14795

22. A. Rahman, S. Pallichankandy, F. Thayyullathil and S. Galadari, Critical role of $\mathrm{H}_{2} \mathrm{O}_{2}$ in mediating sanguinarine-induced apoptosis in prostate cancer cells via facilitating ceramide generation, ERK1/2 phosphorylation, and Par-4 cleavage, Free Radic. Biol. Med. 134 (2019) 527-544; https://doi. org/10.1016/j.freeradbiomed.2019.01.039

23. Y. Zheng, K. Wang, Y. Wu, Y. Chen, X. Chen, C. W. Hu and F. Hu, Pinocembrin induces ER stress mediated apoptosis and suppresses autophagy in melanoma cells, Cancer Lett. 431 (2018) 31-42; https://doi.org/10.1016/j.canlet.2018.05.026

24. M. Gao, W. C. Zhang, Q. S. Liu, J. J. Hu, G. T. Liu and G. H. Du, Pinocembrin prevents glutamateinduced apoptosis in SH-SY5Y neuronal cells via decrease of bax/bcl-2 ratio, Eur. J. Pharmacol. 591 (2008) 73-79; https://doi.org/10.1016/j.ejphar.2008.06.071

25. A. Rasul, F. M. Millimouno, W. Ali Eltayb, M. Ali, J. Li and X. Li, Pinocembrin: a novel natural compound with versatile pharmacological and biological activities, Biomed Res. Int. 2013 (2013) 1-9; https://doi.org/10.1155/2013/379850 